\title{
Ferroptosis and Its Potential Role in Lung Cancer: Updated Evidence from Pathogenesis to Therapy
}

\author{
Kang Chen' \\ Song Zhang ${ }^{2}$ \\ Jinghua Jiao (iD ${ }^{3}$ \\ Shan Zhao ${ }^{4}$ \\ 'Department of Ophthalmology, The \\ First Hospital of China Medical \\ University, Shenyang, Liaoning, People's \\ Republic of China; ${ }^{2}$ China Medical \\ University, Shenyang, Liaoning, People's \\ Republic of China; ${ }^{3}$ Department of \\ Anesthesiology, Central Hospital, \\ Shenyang Medical College, Shenyang, \\ Liaoning, People's Republic of China; \\ ${ }^{4}$ Department of Rheumatic Immunology, \\ The First Hospital of China Medical \\ University, Shenyang, Liaoning, People's \\ Republic of China
}

Correspondence: Jinghua Jiao; Shan Zhao Email jean0905@163.com; zhaoshanI109@I63.com

\begin{abstract}
Lung cancer is characterized by high morbidity and mortality rates, and its occurrence is associated with many types of cell death. As a new form of regulated cell death, ferroptosis is an iron- dependent pattern of cell death and characterized by lethal accumulation of lipid-based reactive oxygen species (ROS), which is different from apoptosis, necrosis and autophagy at both the morphological and biochemical levels. It plays an important role in the development of lung cancer and induction of ferroptosis in lung cancer cells has become a new strategy for anti- lung cancer treatment. However, a few reviews summarized ferroptosis and its role in lung cancer has not been elucidated, and the precise mechanism of ferroptosis modeling lung cancer has not yet been revealed till date. Herein, we review the latest literature on the process of ferroptosis regarding lung cancer, including basic molecular or biology mechanistic studies both in vivo and in vitro, as well as human studies with a more translational or clinical approach. This review provides a practical, concise and updated outline on the mechanisms and therapeutic strategies in lung cancer with ferroptosis alterations. Looking ahead, further studies are required to uncover the possible modulatory relationship between ferroptosis and lung cancer.
\end{abstract}

Keywords: programmed cell death, ferroptosis, biochemical process, lung cancer

\section{Introduction}

Lung cancer is one of the main causes of tumor-related death worldwide, and its high incidence rate and mortality have become the focus of the world, which is responsible for approximately $18.4 \%$ of all cancer mortalities. ${ }^{1,2}$ However, traditional therapies (ie surgery, radiotherapy or chemotherapy), novel molecular targeted therapy or immunotherapy have not brought desirable benefits to patients reaching advanced stages of the disease. ${ }^{3}$ Consequently, clinicians face the dilemma that there is considerable ambiguity in the most appropriate consolidation treatment for patients with advanced stages of lung cancer. Therefore, further exploring the occurrence and development mechanisms of lung cancer, discovering new therapeutic targets, and finding effective therapeutic methods and drugs should be urgently designed and implemented.

Cell death is important for mammalian development and homeostasis and is fully integrated with the physiological function and pathological state of an organism. ${ }^{4}$ The orchestration of cell death both spatially and/or temporally is critical for the development of various human diseases. ${ }^{5}$ Regarding most of the different cells in the body, cell death can be conventionally divided into four distinctive types: apoptosis, necrosis, autophagy, and pyroptosis. ${ }^{6}$ In 2012, a new non-apoptotic cell death pattern caused 
by an iron-dependent lipid peroxidation injury was presented and named "Ferroptosis". 7

Ferroptosis is a cell death caused by cell membrane damage due to glutathioneperoxidase (GPX) activity failure and intracellular lipid peroxide, accompanied by the irondependent production of reactive oxygen species (ROS) ${ }^{7,8}$ Its morphology, genetics and biochemical characteristics are significantly different from apoptosis, necrosis, autophagy and pyroptosis (Table 1). ${ }^{9}$ Morphologically, ferroptosis occurs mainly in cells as reduced mitochondrial volume, increased bilayer membrane density and reduction or disappearance of mitochondrial cristae, without nuclear concentration and chromatin marginalization. ${ }^{10}$ Generally, the mitochondrion can regulate ROS generation, ferroptosis and cell cycle, and it has been implicated in various malignancies including lung cancer. Furthermore, irradiation and hypoxia promote mitochondrial stress pathways activity to survive the harsh environment. Against normal cells, both the consumption of ROS and irons are elevated in tumor cells as a consequence of increased metabolic rate. ${ }^{11,12}$ Consequently, the above changes inhibit ferroptosis in tumor cells. At present, many studies on ferroptosis and lung cancer have made certain progress. Of which, ferroptosis inducers are used as traditional treatment programs and new adjuvants have been shown to be effective in the treatment of lung cancer. Therefore, inducing ferroptosis in lung cancer cells has become a new anti-cancer treatment strategy. ${ }^{13,14}$ Generally, ferroptosis plays an important role in the development and therapeutics of lung cancer, which we will describe in detail in the following sections.

\section{Mechanism of Ferroptosis}

To date, the major biochemical processes involved in ferroptosis can be simply comprised of four steps, ie, (i) inactivation of cysteine/glutathione antiporter system $\mathrm{Xc}^{-}(\mathrm{xCT})$, (ii) depletion of glutathione and lipid repair enzyme (glutathione

Table I Cell Morphology, Biochemical Features, and Key Regulators of Necrosis, Autophagy, Apoptosis, Pyroptosis, Ferroptosis

\begin{tabular}{|c|c|c|c|}
\hline $\begin{array}{l}\text { Type of } \\
\text { Cell } \\
\text { Death }\end{array}$ & Identification & Cell Morphology & Biochemical Features \\
\hline Necrosis & $\begin{array}{l}\text { Traumatic cell death due to mechanical } \\
\text { shear stress or other physicochemical } \\
\text { factors. }\end{array}$ & $\begin{array}{l}\text { Mitochondria swells in cytoplasm, forming } \\
\text { vesicles of different sizes, and } \\
\text { mitochondrial cristae break until they } \\
\text { disappear. Cytoplasm can lose water, and } \\
\text { cell volume is reduced. }\end{array}$ & Necrosome \\
\hline Autophagy & $\begin{array}{l}\text { A process of engulfing one's own } \\
\text { cytoplasmic proteins or organelles and } \\
\text { coating them into vesicles, fusing with } \\
\text { lysosomes to form autophagic lysosomes, } \\
\text { and degrading the contents it } \\
\text { encapsulates. }\end{array}$ & $\begin{array}{l}\text { Autolysosome accumulation, cytoplasmic } \\
\text { vacuolization. }\end{array}$ & $\begin{array}{l}\text { Conversion from light chain } 3 \text { (LC3)-I to } \\
\text { LC3-II, degradation of p62Lck, Beclin-I } \\
\text { dissociation from Bcl-2/XL }\end{array}$ \\
\hline Apoptosis & $\begin{array}{l}\text { A morphologically distinct form of } \\
\text { programmed cell death that is mediated } \\
\text { by a number of proteases that cleave their } \\
\text { target proteins at specific aspartate } \\
\text { residues. }\end{array}$ & $\begin{array}{l}\text { Plasma membrane blebbing; reduction of } \\
\text { cellular and nuclear volume; nuclear } \\
\text { fragmentation; and chromatin } \\
\text { condensation }\end{array}$ & $\begin{array}{l}\text { Activation of caspases and proapoptotic } \\
\text { Bcl-2 family proteins, oligonucleosomal } \\
\text { DNA fragmentation, exposure of Plasma } \\
\text { membrane rupture, dissipation of } \\
\text { dissipation. }\end{array}$ \\
\hline Pyroptosis & $\begin{array}{l}\text { A unique form of programmed cell death } \\
\text { mediated by the gasdermin family of } \\
\text { proteins and causes lytic cell death and } \\
\text { release of proinflammatory cytokines. }\end{array}$ & $\begin{array}{l}\text { Cellular swelling and lysis, release } \\
\text { inflammatory factors. }\end{array}$ & Inflammasome \\
\hline Ferroptosis & $\begin{array}{l}\text { A type of regulated cell death caused by } \\
\text { an excess iron-dependent accumulation of } \\
\text { lipid peroxides and is morphologically and } \\
\text { biochemically distinct from other types of } \\
\text { cell death. }\end{array}$ & $\begin{array}{l}\text { Small mitochondria with a condensed } \\
\text { mitochondrial membrane, vanishing or } \\
\text { reduction of mitochondria crista, and } \\
\text { rupture of outer mitochondrial membrane }\end{array}$ & $\begin{array}{l}\text { Iron loading, ROS accumulation, System } \\
\text { Xc- inhibition with reduced GSH, GPX } 4 \\
\text { inhibition }\end{array}$ \\
\hline
\end{tabular}




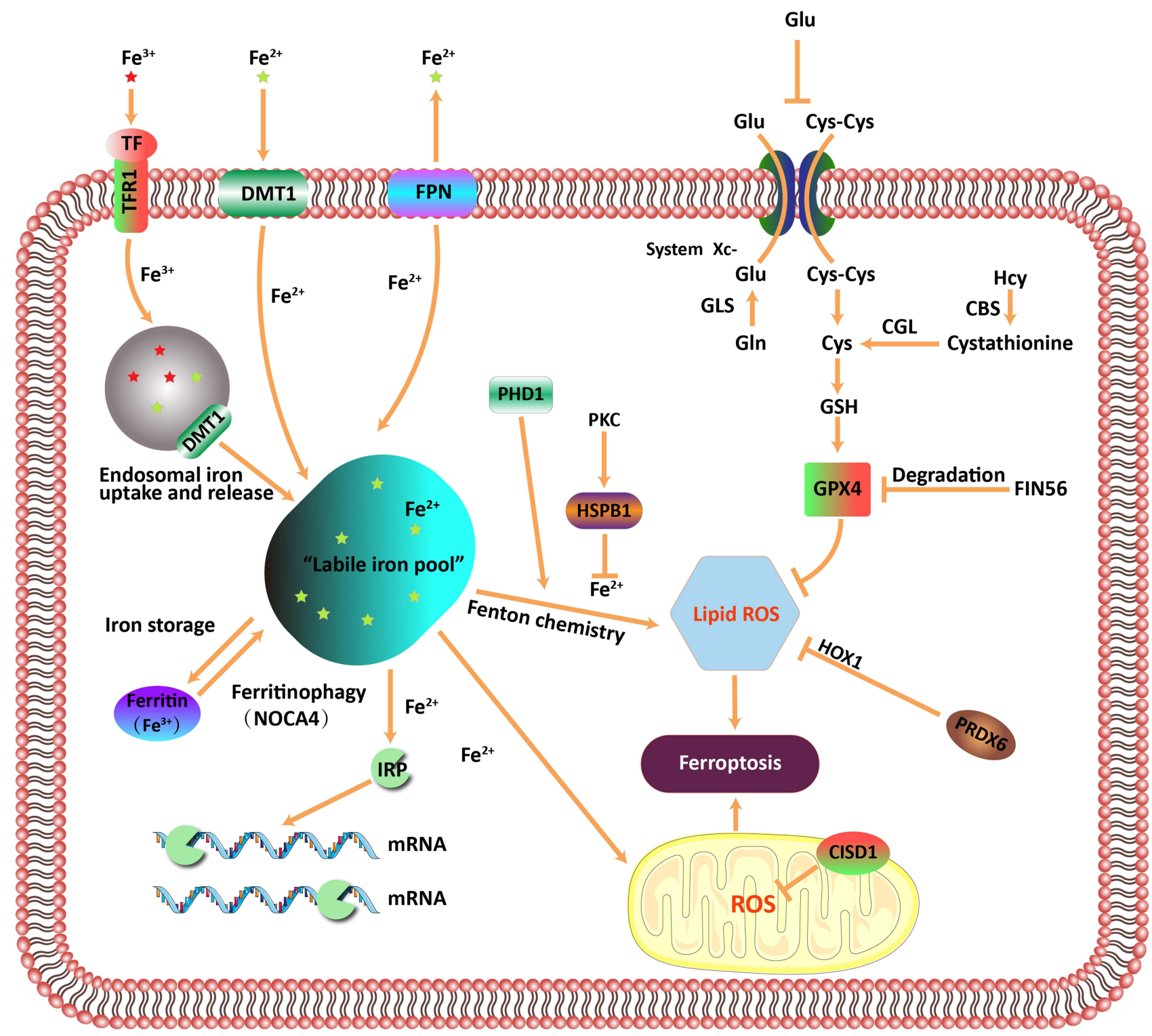

Figure I Main regulatory mechanism of ferroptosis. Iron homeostasis, and lipid peroxidation metabolism are core regulators of ferroptosis.

Abbreviations: TFRI, transferrin receptor I; DMTI, divalent metal ion transporter I; FPN, ferroportin; IRP, iron regulatory protein; PHDI, prolyl hydroxylase domain protein I; HSPBI, heat shock protein $\beta$-I; FIN56, specific iron death inducer; System Xc-, cystine/glutamate transporter; Cys, cysteine; Glu, glutamic; Gln, glutamine; GLS, glutaminase; GSH, glutathione; GPX4, glutathione peroxidase 4; Hcy, homocysteine; CGL, cystathionine- $\gamma$-lyase; HOXI, heme oxygenase-I; PRDX6, peroxide reduction enzyme-6; CISDI, CDGSH iron sulfur domain I.

peroxidase 4, GPX4), (iii) lipid peroxidation and (iv) excess iron and accumulation of ROS in cells. ${ }^{15-17}$ The main regulatory mechanism of ferroptosis is shown in Figure 1.

\section{Cysteine/Glutathione Antiporter System}

The cysteine/glutathione antiporter, also named system $\mathrm{Xc}^{-}$, as a trans-membrane protein, is an important intracellular antioxidant element. System $\mathrm{Xc}^{-}$is composed of the light-chain SLC7A11 (xCT) and the heavy-chain SLC3A2 (CD98hc or 4F2hc) and its functions are as raw materials for the synthesis of glutathione (GSH). ${ }^{18}$ As its main functional subunit, SLC7A11 encodes a cystine transporter also known as $\mathrm{xCT}$, which is highly specific to cystine and glutamic acid (Glu), responsible for the major transport activity. Additionally, CD98hc as a companion protein maintains the stability of $\mathrm{xCT}$ protein. ${ }^{19}$ System $\mathrm{Xc}^{-}$regulates the 1:1 exchange of extracellular cystine for intracellular glutamic acid in and out of cells. $^{20}$ Interestingly, Glu, cysteine (Cys), and glycine (Gly) under the catalysis of glutamate cysteine ligase (GCL) and glutamylcysteine synthetase (GCS) generated GSH. On the other hand, GPXs are kinds of highly 
conservative enzymes on evolution, which are essential components of cellular detoxification systems that defend cells against ROS. ${ }^{21}$ To the best of our knowledge, GPX4 is the core controlling factor in ferroptosis, and the intracellular GSH content directly affects GPX4 enzyme activity. $^{22}$ Currently, many small-molecule inducers, namely ferroptosis-inducing agents (FINs), are identified as ferroptosis-inducing compounds. Like ras selective lethal 3 (RSL3), FIN56 inhibits GPX4 activity without GSH depletion. ${ }^{23,24}$ Inactivation of GPX4 by GSH depletion is found to trigger ferroptosis. ${ }^{25,26}$ Thus, elucidation of the factors that underlie the sensitivity of a given cell type to ferroptosis is crucial to understand the physiological and pathophysiological manifestation of ferroptosis and how it may be exploited for the management of cancer.

\section{Iron Metabolism}

Iron (Fe) exists in two oxidation states including the ferrous cation $\left(\mathrm{Fe}^{2+}\right)$ and ferric cation $\left(\mathrm{Fe}^{3+}\right)$. Non-haem $\mathrm{Fe}$ in food is mainly in the form of $\mathrm{Fe}^{3+}$, which is reduced back to $\mathrm{Fe}^{2+}$ by $\mathrm{Fe}$ reductase, such as duodenal cytochrome b (Dcytb) ${ }^{27}$ and antioxidant enzyme heme oxygenase-1 (HO-1) in the intestine. ${ }^{28}$ Dietary Fe enters the intestinal epithelium cells (IECs) via the brush-border transporter divalent metal transporter 1 (DMT1) and exits through the basolateral membranes. ${ }^{29}$ Overexpression of DMT1 promoted cell ferroptosis, while knockdown of DMT1 significantly inhibited the ferroptosis. ${ }^{30}$ Meanwhile, ferrous cation $\left(\mathrm{Fe}^{2+}\right)$ absorbed by IECs is transported by the action of ferroportin-1 (FPN1) on the extracellular side of the membrane. An oxidation of $\mathrm{Fe}^{2+}$ into $\mathrm{Fe}^{3+}$ by ceruloplasmin (CP), hephaestin (HP) and poly(rC)-binding protein 2 (PCBP2), which combines with transferrin (Tf) to form the Tf (with loading two $\mathrm{Fe}^{3+}$ ) complex. ${ }^{31}$ Then, Tf$\mathrm{Fe}^{+}$binds to the transferrin receptor (TfR) forming $\mathrm{Fe}^{3+}$ $\mathrm{Tf} / \mathrm{TfR}$ complex on the cell membrane and internalizes to cell as endosomes, ${ }^{32}$ whereas $\mathrm{Fe}^{3+}$ is released and subsequently transported by six-transmembrane epithelial antigen of the prostate 3 (STEAP3) reduced to $\mathrm{Fe}^{2+}$, where iron enters the cytoplasm via DMT1 on the endosomal membrane. ${ }^{33,34}$

Oxidation of excess ferrous iron to the ferric state in the cell cytoplasm is known as unstable iron pool, which has metabolic activity and plays a crucial role in a variety of biological functions, such as ferroptosis. ${ }^{35}$ Cellular iron homeostasis needs to be tightly regulated by balancing its uptake, transport, and storage. Intracellular iron deficiency can lead to insufficient energy production, whereas iron overload triggers ROS formation via the Fenton reaction, which begins with the oxidation of the ferrous ion $\left(\mathrm{Fe}^{2+}\right)$ to the ferric ion $\left(\mathrm{Fe}^{3+}\right)$ in the presence of hydrogen peroxide, which acts as an oxidizing agent $\left(\mathrm{Fe}^{2+}+\right.$ $\left.\mathrm{H} 2 \mathrm{O} 2 \rightarrow \mathrm{Fe}^{3+}+\cdot \mathrm{OH}+\mathrm{OH}^{-}\right) .{ }^{36}$ Through Fenton reaction, a large number of hydroxyl radicals are produced, triggering a strong oxidative stress reaction, producing a large number of ROS and inducing ferroptosis. ${ }^{37}$ Thereby, iron is an essential element for ferroptosis, and iron metabolism is a necessary process for ferroptosis.

\section{Lipid Metabolism}

Ferroptosis is also driven by iron-dependent lipid peroxidation. ${ }^{38}$ Lipid peroxidation refers to the process in which oxidants obtain an unstable hydrogen atom from the diallyl methyl phosphate of polyunsaturated fatty acids (PUFAs) and generates a large number of free radical lipid peroxidation and hydrogen peroxide content. ${ }^{25}$ The content of intracellular PUFAs determines the degree of lipid peroxidation in cells and its sensitivity to ferroptosis. ${ }^{39}$ Recent studies have revealed that many factors or signaling molecules participate in the regulation of fatty acid synthesis, such as glutamine (Gln), citrate synthase (CS) and acetyl-CoA carboxylase (ACC), participating in the regulation of ferroptosis by mediating lipid oxidation. ${ }^{40-42}$ However, the exact mechanism of these factors' role in ferroptosis remains unclear and will be an exciting topic for future studies.

So far, many factors participate in the production of lipid peroxides such as Acyl-CoA synthetase long-chain family 4 (ACSL4), lysophosphatidylcholine acyltransferase 3 (LPCAT3), and lipoxygenases (LOXs). ${ }^{43}$ ACSL4 is a member of the long-chain acyl coenzyme A synthase family (ACSLs), which is a class of essential enzymes involved in fatty acid metabolism. ${ }^{44}$ LPCAT3 is a protein that catalyzes the reacylation of lysophospholipids to phospholipids. Li et al revealed that the knockdown of LPCAT3 activity has been shown to increase intracellular lysophospholipid levels and promote very low-density lipoprotein secretion in hepatocytes. ${ }^{45}$ LOXs (the 15LOX-1 isoform, in particular) have been implicated as a key regulator in ferroptotic cell death. ${ }^{46,47}$ Depending on cellular contexts, ACSL4 and LPCAT3 activate free long-chain polyunsaturated fatty acids, promote the conversion of lysophosphatidylcholine (LPC) into lecithin, and participate in the synthesis of phospholipid of oxidized cell membrane, thus mediating the fereoptosis process. On 
the other hand, ACSL4 esterify mainly arachidonic acid (AA) into acyl-CoA, for biosynthesis of polyunsaturated fatty acids required for fatty acid oxidation and ferroptosis. $^{48}$ The absence of CoA leads to a decrease in lipid peroxidation substrates, which declines the level of ferroptosis. Yang et al have demonstrated that cells are resistant to ferroptosis when LOXs activities are knocked down by small interfering RNA (siRNA). ${ }^{47}$ LOXs are not only essential for the execution of ferroptosis but may also play a key role in its initiation by contributing to the lipid hydroperoxides, which promote lipid autoxidation. ${ }^{49}$

\section{The Role of Ferroptosis in Lung Cancer}

Based on the theories above, ferroptosis is a form of irondependent cell death characterized by the accumulation of lipid peroxides, and it plays a dual role in tumor occurrence. Generally speaking, many drugs can induce ferroptosis to suppress lung cancer cell growth by causing cysteine depletion or by inactivating GPXs. Additionally, ferroptosis could evoke immunosuppression to promote tumor growth of NSCLC by mediating the inflammatory responses. ${ }^{50}$ Herein, combining ferroptosis inhibitors with immunotherapy may be a novel strategy for lung cancer therapy.

\section{Ferroptosis and the Development of Lung Cancer Iron lon}

Epidemiological and laboratory findings confirmed that iron intake is associated with the development of lung cancer. In a previous prospective population-based cohort study using the Rotterdam dataset, researchers found that dietary higher intake of iron was associated with a decreased risk of lung cancer after adjusting for potential variables. $^{51}$ These findings are in agreement with the results from another prospective, National Institutes of Health-American Association of Retired Persons (NIHAARP) Diet and Health Study with 7052 lung cancer cases, which showed that a higher intake of dietary iron was associated with a significantly reduced risk of lung cancer (13\%) after mean follow-up of 7 years. ${ }^{52}$ A risk reduction ranging from $19 \%$ to $34 \%$ for total iron intake was found in a case-control study with 1139 cases and people with low $\mathrm{Fe}^{+2}$ suboptimal DNA repair capacity (DRC) may have approximately two-fold risk for lung cancer compared with those with high $\mathrm{Fe}^{+2}$ proficient DRC. ${ }^{53}$ In a Massachusetts hospital-based case-control study involving 923 cases and 1125 healthy controls, dietary iron intake may play an important role in the development of lung cancer, whereas heme iron was associated with a decreased risk. ${ }^{54}$ Mechanically, minerals including iron are essential dietary intakes for maintaining the integrity of DNA by preventing oxidative DNA damage. ${ }^{55}$ Moreover, iron deficiency or overload may lead to oxidative DNA damage, ${ }^{56}$ which can further predispose to cancer development. ${ }^{57}$ Similar findings from epidemiological evidence have shown that DNA repair capacity is associated with increased lung cancer risk. ${ }^{58,59}$ Previous laboratory data have identified excessive iron can induce apoptosis, necrosis and ferroptosis. Erastin, first discovered as an inducer of ferroptosis, promotes iron-dependent cell death accompanied by antioxidant depletion caused by cystine glutamate antiporter inhibition. ${ }^{60}$ In BALB/c (nu/ $n u)$ mice model of lung cancer, overexpression of transferrin receptor 1 (TFR-1) can accelerate the iron absorption rate of lung cancer cells, promote tumor growth, and shorten the mice survival time. ${ }^{61}$ Moreover, heat shock protein B 1 (HSPB1) is a negative regulator of ferroptosis as it inhibits the accumulation of iron by inhibiting TFR1 expression. ${ }^{62}$ Noteworthy, although iron can catalyze the production of ROS through Fenton reaction and modulate ferroptosis, its underlying mechanism for lung cancer is still unknown. Following the publication of the above paper, there is no report on whether and how normal cells also rely on iron to cause death, or whether their changes are significantly different from lung cancer cells.

\section{SLC7AII}

SLC7A11, as a potential biologic marker, is highly expressive in non-small cell lung cancer (NSCLC). ${ }^{63}$ SLC7A11 can promote metastasis as well as proliferation of lung cancer cells both in vitro and in vivo, and downregulating SLC7A11 can inhibit metastasis as well as proliferation of lung cancer cells. ${ }^{64,65}$ Furthermore, SLC7A11 overexpression could restore the REDOX homeostasis under stress by mediating cystine uptake, reducing ROS production and promoting the proliferation and migration abilities of human lung adenocarcinoma (A549). ${ }^{66}$ On the contrary, down-regulation of SLC7A11 gene expression by siRNA can induce ROS accumulation, and contribute to ferroptotic cell death and inhibit A549 cell proliferation. ${ }^{67}$ In patients with The Kirsten Rat Sarcoma (KRAS)-mutant lung adenocarcinoma (LUAD), SLC7A11 was overexpressed and positively related to the progression of tumor progression. Correspondingly, 
a potent SLC7A11 inhibitor, HG106, markedly decreased cystine uptake and intracellular glutathione biosynthesis. Furthermore, inhibiting SLC7A11 expression by HG106 can reduce intracellular cystine uptake, inhibit intracellular GSH biosynthesis, and, thus, significantly inhibit tumor growth and metastasis in vitro, prolong the survival time of mice with lung cancer, and exhibit selective cytotoxicity toward KRAS-mutant cells by increasing oxidative stressmediated cell apoptosis in vivo. ${ }^{68}$ Overall, these findings reveal that KRAS-mutant LUAD cells are sensitive to SLC7A11 inhibition, bringing potential therapeutic approaches for this currently incurable disease.

\section{The Family of Glutathione Peroxidase}

The family of GPXs, as an antioxidant enzyme in human tissues, has been identified to play an important role in the development of cancers. Lung cancer cell lines also showed high expression of GPXs, such as GPX8 and GPX4. Clinically, GPX8 expression in NSCLC samples was statistically much higher than that in the nontumorous tissues. The high expression of GPX8 is correlated with the worse clinical prognosis of NSCLC patients. Mechanically, these effects may be due to GPX8 inhibiting the apoptosis of tumor cells and promoting its migration and invasion. ${ }^{69}$ Recently, Wang and co-researchers found that the promoter region of GPX4 could be bound by cyclic adenosine monophosphate (cAMP) response element-binding (CREB) protein, and this binding could be enhanced by E1A binding protein P300 (EP300). Furthermore, CREB, GPX4, EP300 and 4-Hydroxynonenal (4-HNE) were closely related to cancer development, such as proliferation, migration, invasion and angiogenesis. Hence, targeting this CREB/EP300/ GPX4/4-HNE pathway may provide a new strategy for treating LUAD. $^{70}$ Additionally, the overexpression of GPX4 can promote the proliferation of the lung cancer cell and resist ferroptosis, whereas RSL3 inhibits the activity of GPX4 and limits proliferation, migration, and invasion of A549 cells. ${ }^{71}$ Notably, ferrostatin-1 (Fer-1) which is an inhibitor of ferroptosis, can reverse the above phenomenon. ${ }^{72}$ This means that inhibiting GPX4 can induce ferroptosis of lung cancer cell, and it may be a new therapeutic regimen under investigation that would benefit patients with lung cancer.

\section{Ferroptosis Suppressor Protein I}

Ferroptosis suppressor protein 1 (FSP1) is a ferroptosis inhibitor, which is independent of classical GPX4 signaling pathway and can suppress ferroptosis by ubiquinone (also known as coenzyme Q10, CoQ10). ${ }^{22}$ After down-regulating GPX4 gene expression, FSP1 is decorated with cardamom acylation, by regulating $\mathrm{NAD}(\mathrm{P}) \mathrm{H}$ to reduce CoQ10, generating lipophilic free radicals to capture free antioxidants (radical-trapping antioxidant, RTA) to prevent lipid peroxidation, so as to inhibit ferroptosis. ${ }^{22}$ FSP1 expression led to a higher degree of resistance in lung cancer cells. On the contrary, FSP1 inhibitor (iFSP1) can reverse ferroptosis' resistance caused by FSP1, increase the sensitivity of lung cancer cells to ferroptosis, and promote ferroptosis in lung cancer cells. ${ }^{22}$ At present, the research on FSP1 is still in the embryonic stage, and further research is needed.

\section{Ferroptosis and p53 in Lung Cancer}

Recently, emerging evidence suggests that p53 could induce ferroptosis. Meng et al revealed that levobupivacaine, a widely used local anesthetic, could inhibit the proliferation and induce the apoptosis of NSCLC cells. Moreover, the medication of levobupivacaine enhanced the erastin-induced inhibition of proliferation of NSCLC cells. Mechanically, the medication of levobupivacaine could promote the levels of ROS, iron, and $\mathrm{Fe}^{2+}$ in NSCLC cells and induce ferroptosis by regulating p53 expression in NSCLC cells. Similar results were also found in vivo, where the treatment of levobupivacaine significantly repressed the tumor growth of NSCLC cells. $^{73}$

\section{Ferroptosis in EGFR-Activating Mutant Lung Adenocarcinoma}

The discovery of activating mutations in the epidermal growth factor receptor (EGFR) gene and the development of EGFR tyrosine kinase inhibitors (EGFR-TKIs) have led to a paradigm shift in the treatment of lung cancer. ${ }^{74}$ Currently, emerging evidence suggests that ferroptosis can be therapeutically exploited for the treatment of solid tumors and ferroptosis-inducing therapy shows promise in EGFR-activating mutant lung cancer cells that display intrinsic or acquired resistance to EGFR-TKI. ${ }^{75}$

\section{Ferroptosis and Drug Resistance of Lung Cancer}

Cisplatin (DDP) elevates the activities of malondialdehyde (MDA) as well as ROS, promotes the expression of heme oxygenase 1 (HO-1) and $\mathrm{NAD}(\mathrm{P}) \mathrm{H}$ quinone oxidoreductase 1 (NQO-1), and also induces ferroptosis of lung 
cancer cells by regulating lipid peroxidation. ${ }^{76-78}$ Activation of $\mathrm{Nrf} 2 / \mathrm{xCT}$ pathway is one of the main mechanisms of Cisplatin resistance in NSCLC cells. ${ }^{79}$ Both erastin and sorafenib significantly induce ferroptosis and decrease cell activity, and enhance the sensitivity of NSCLC cells to DDP by inhibiting the expression of $\mathrm{xCT}$, and Nrf2 downstream targets. ${ }^{79}$ In contrast, the overexpression of SLC7A11 can enhance DDP resistance in lung cancer cells. ${ }^{66,80}$ The expression of SLC7A11 was associated with the efficacy of many candidate anticancer drugs, of which SLC7A11 was positively correlated with the efficacy of 39 drugs and negatively correlated with the efficacy of 296 drugs, suggesting that SLC7A11 could be used as a predictor factor of GSH-mediated anticancer drug resistance to predict the sensitivity of various chemical drugs. ${ }^{81}$

\section{Ferroptosis and Radiation Resistance}

Under harsh environmental conditions such as ionizing radiation (IR), it induces not only ROS but also the expression of ACSL4 in NSCLC cells, resulting in elevated lipid peroxidation and ferroptosis. ${ }^{82}$ Additionally, IR also induces the expression of ferroptosis inhibitors such as SLC7A11 and GPX4, as an adaptive response. Interestingly, IR deficiency-induced SLC7A11 expression activates the radioresistance by inhibiting ferroptosis. ${ }^{82}$ Inactivating SLC7A11 or GPX4 with ferroptosis inducers (FINs) sensitizes radioresistant H460, A549, and H1299 cell lines and xenograft tumors to IR. Furthermore, radiotherapy induces ferroptosis in cancer patients, and increased ferroptosis correlates with better response and longer survival to radiotherapy in esophageal cancer patients. $^{82}$ There is a previously unrecognized link between IR and ferroptosis, which indicates that further exploration of the combination of radiotherapy and FINs in cancer treatment is warranted.

\section{Ferroptosis and Potentiate the Effects of Immunotherapy}

$\mathrm{T}$ cell mediated cellular immunity plays a key role in the development of tumor. In the process of immunotherapy, activated $\mathrm{CD} 8(+)$ effector T-cells can enhance the specific lipid peroxidation of ferroptosis in tumor cells. ${ }^{83}$ Otherwise, the activation of ferroptosis may help to the anti-tumor effect of immunotherapy. CD8(+) effector T-cells release IFN- $\gamma$ under expression, inhibit cystine uptake, and promote lipid peroxidation and ferroptosis. $^{83,84}$

Exhaustion of intracellular cystine or blocking of antiprogrammed cell death protein 1/programmed death ligand 1 (anti-PD-1/PD-L1) immune checkpoint significantly enhanced $\mathrm{T}$ cell-mediated anti-tumor immunity and induced the ferroptosis of tumor cell. ${ }^{85}$ At the same time, clinical data showed that among patients with melanin, the expression of cystine associated transporters SLC7A11 and SLC3A2 was negatively correlated with the number of $\mathrm{CD} 8+\mathrm{T}$ cells, IFN- $\gamma$ expression level and patient prognosis. $^{86}$ Despite recently targeting lung cancer, the relationship between $\mathrm{T}$ cell and ferroptosis is uncertain. However, it is not difficult to find that $\mathrm{T}$ cell promotes the ferroptosis of lung cancer cell is a potential treatment and can enhance the treatment efficiency of immunotherapy. Thereby, it is inevitable that continuing research in this field will further elucidate the physiological and pathological roles of ferroptosis, leading to the development of translational anticancer strategies. Research into biomarkers to precisely trace ferroptosis in patients with cancer, and the development and subsequent application of novel ferroptosis-based therapies will be of critical importance in the next few years.

\section{Targeting Ferroptosis Inducers for Lung Cancer Therapy}

Emerging findings reveal the crucial contribution of ferroptosis as a potential target for lung cancer therapy and ferroptosis inducers including small molecules and nanomaterials have been developed. ${ }^{87}$ Nanotechnology applications have attracted much attention with specific physicochemical properties recently, and the detailed information on those previously reported nanoparticles is shown in Table 2.

Most nanomaterials such as iron-based nanoparticles are based on Fenton reaction. In order to open up a new avenue for developing multifunctional advanced new materials for cancer therapy, Yao et al prepared a tumor-targeted nanoparticle named FeCO-DOX@MCN (a multifunctional CO/ thermo/chemotherapy) nanoplatform targeting near-infrared (NIR) light and convert it into ample heat to trigger $\mathrm{CO}$ release and could also release doxorubicin (DOX) in the acidic tumor microenvironment. Its mechanisms on anticancer included iron loading, ROS level increase, GSH depletion, and GPX4 inactivation. In addition, the generated $\mathrm{CO}$ molecules successfully increased tumor sensitivity to 
Table 2 Nanoparticle Inducers in Ferroptosis for Lung Cancer

\begin{tabular}{|c|c|c|c|c|}
\hline $\begin{array}{l}\text { Nanoparticle } \\
\text { Inducers }\end{array}$ & Mechanism & Model & Advantages & Reference \\
\hline $\begin{array}{l}\text { FeCO-DOX } \\
@ M C N\end{array}$ & $\begin{array}{l}\text { Iron loading, ROS level increase, GSH } \\
\text { depletion, GPX4 inactivation }\end{array}$ & $\begin{array}{l}\text { In-vitro: MCF-7, } \\
\text { A549, HeLa; In-vivo } \\
\text { : MCF-7 }\end{array}$ & $\begin{array}{l}\text { Under the guidance of photoacoustic } \\
\text { imaging, the FeCO-DOX@MCN } \\
\text { nanoplatform demonstrates high treatment } \\
\text { efficacies in vitro and in vivo by } \\
\text { combination of chemotherapy, } \\
\text { photothermal therapy and gas therapy. }\end{array}$ & [88] \\
\hline $\begin{array}{l}\text { Exosome-like } \\
\text { nanovesicles } \\
\text { (eNVs-FAP) }\end{array}$ & $\begin{array}{l}\text { eNVs-FAP vaccine-activated cellular } \\
\text { immune responses could promote tumor } \\
\text { ferroptosis by releasing interferon-gamma } \\
\text { (IFN- } \gamma \text { ) from CTLs and depleting FAP } \\
+ \text { CAFs. }\end{array}$ & $\begin{array}{l}\text { Colon, melanoma, } \\
\text { lung, and breast } \\
\text { cancer models }\end{array}$ & $\begin{array}{l}\text { eNVs-FAP showed excellent antitumor } \\
\text { effects in a variety of tumor-bearing mouse } \\
\text { models. }\end{array}$ & [89] \\
\hline $\begin{array}{l}\text { Phenylboronate } \\
\text { ester (PBE) }\end{array}$ & $\begin{array}{l}\text { The nanoparticles can perform acid- } \\
\text { activatable photodynamic therapy by } \\
\text { protonation of the ionizable core, and } \\
\text { significantly recruit tumor-infiltrating } \\
\text { T lymphocytes for interferon gamma } \\
\text { secretion, and thus sensitize the tumor } \\
\text { cells to RSL-3-inducible ferroptosis. }\end{array}$ & $\begin{array}{l}\text { Lung metastasis of } \\
4 \mathrm{TI} \text { breast tumors }\end{array}$ & $\begin{array}{l}\text { The combination of nanoparticle-induced } \\
\text { ferroptosis and blockade of programmed } \\
\text { death ligand I efficiently inhibits growth of } \\
\text { BI6-FIO melanoma tumor and lung } \\
\text { metastasis of } 4 \mathrm{TI} \text { breast tumors, } \\
\text { suggesting the promising potential of } \\
\text { ferroptosis induction for promoting cancer } \\
\text { immunotherapy. }\end{array}$ & [90] \\
\hline $\begin{array}{l}\text { Zero-valent- } \\
\text { iron } \\
\text { nanoparticle } \\
\text { (ZVI-NP) }\end{array}$ & $\begin{array}{l}\text { ZVI-NP caused mitochondria dysfunction, } \\
\text { intracellular oxidative stress, and lipid } \\
\text { peroxidation, leading to ferroptotic death } \\
\text { of lung cancer cells. Degradation of NRF2 } \\
\text { by GSK3/ } \beta-T r C P \text { through AMPK/mTOR } \\
\text { activation was enhanced in such cancer- } \\
\text { specific ferroptosis. }\end{array}$ & $\begin{array}{l}\text { Immunocompetent } \\
\text { mice and } \\
\text { humanized mice. }\end{array}$ & $\begin{array}{l}\text { This dual-functional nanomedicine } \\
\text { established an effective strategy to } \\
\text { synergistically induce ferroptotic cancer } \\
\text { cell death and reprogram the } \\
\text { immunosuppressive microenvironment, } \\
\text { which highlights the potential of ZVI-NP as } \\
\text { an advanced integrated anti-cancer } \\
\text { strategy. }\end{array}$ & [91] \\
\hline $\begin{array}{l}\text { Citric acid iron } \\
\text { oxide } \\
\text { nanoparticles } \\
\text { (IONPs-Ac) }\end{array}$ & $\begin{array}{l}\text { It displayed important cytotoxicity in } \\
\text { a human lung cancer cells model (A549 } \\
\text { cells), even at low concentrations, whereas } \\
\text { free IONPs-Ac displayed adequate } \\
\text { biocompatibility. }\end{array}$ & A549 cells & $\begin{array}{l}\text { It was possible to infer that ferri-liposomes } \\
\text { were able to induce A549 tumor cells } \\
\text { death through apoptosis/ferroptosis } \\
\text { processes, evidenced by a significant } \\
\text { reduction of the mitochondrial membrane } \\
\text { potential. }\end{array}$ & [92] \\
\hline $\begin{array}{l}\text { Erastin/ } \\
\text { MTIDP@FA- } \\
\text { LPs (E/M@FA- } \\
\text { LPs) }\end{array}$ & $\begin{array}{l}\text { Folate (FA)-modified liposome (FA-LP) } \\
\text { nanoparticles for targeted co-delivery of } \\
\text { erastin and MTIDP to enhance the } \\
\text { bioavailability and the efficiency of the } \\
\text { drug/gene combination. }\end{array}$ & $\begin{array}{l}\text { A549 and HI299 } \\
\text { cells }\end{array}$ & $\begin{array}{l}\text { Identify a novel strategy to elevate erastin- } \\
\text { induced ferroptosis in NSCLCs acting } \\
\text { through the MTIDP/miR-365a-3p/NRF2 } \\
\text { axis. }\end{array}$ & [93] \\
\hline
\end{tabular}

chemotherapeutics through the ferroptosis pathway. It is worth noticing that under the guidance of photoacoustic imaging, the FeCO-DOX@MCN nanoplatform demonstrates high efficacy both in vitro and in vivo through a combination of chemotherapy, photothermal therapy and gas therapy. This multifunctional platform with excellent antitumor efficacy has great potential in precision cancer therapy. ${ }^{88}$

\section{Conclusions and Perspectives}

Ferroptosis, as a newly discovered form of cell death, has a unique advantage and large potential in the treatment of tumor. The sensitivity of many aggressive and drugresistant cancer cells to ferroptosis and the FDA approval of altretamine, sorafenib and silica nanoparticles as ferroptosis inducers for tumor therapy have generated high expectations in the treatment of ferroptosis. Although the 
research on ferroptosis has made great progress in recent years, there are still some problems to be solved, such as the particularity of ROS in ferroptosis and the specific role of ferroptosis in immunotherapy. In addition, there were significant differences in the sensitivity of cells to ferroptosis among different tissues, and there were also significant differences among individuals in the sensitivity to ferroptosis inducers, such as erastin and sorafenib. Therefore, it is of great significance to find biological indicators that can reflect the sensitivity of cells and individuals to ferroptosis and to discover new ferroptosis inducers for improving the understanding of ferroptosisrelated diseases and the diagnosis and treatment level of lung cancer. Ferroptosis will become a new strategy for cancer treatment, breaking the current dilemma of lung cancer treatment and bringing benefits to lung cancer patients.

\section{Acknowledgments}

We thank Prof Lei Liu for discussion and comments.

\section{Funding}

The authors would like to acknowledge financial support from the National Natural Science Foundation of China (Grant No. 82003882). The Natural Science Foundation of Liaoning Province (Grant No. 2019-ZD-0339). Shenyang Young and Middle-aged Science and Technology Innovation Talent Support Program (Grant No. RC200528). The Natural Science Foundation of Liaoning Province (Grant No. 19-112-4-096). All authors approved the final version of the article.

\section{Disclosure}

The authors declare that there is no conflict of interest in this work.

\section{References}

1. Bray F, Ferlay J, Soerjomataram I, Siegel RL, Torre LA, Jemal A. Global cancer statistics 2018: GLOBOCAN estimates of incidence and mortality worldwide for 36 cancers in 185 countries. CA Cancer J Clin. 2018;68(6):394-424. doi:10.3322/caac.21492

2. Abdulbaqi IM, Assi RA, Yaghmur A, et al. Pulmonary delivery of anticancer drugs via lipid-based nanocarriers for the treatment of lung cancer: an update. Pharmaceuticals. 2021;14(8):725. doi:10.3390/ ph14080725

3. Zukotynski KA, Hasan OK, Lubanovic M, Gerbaudo VH. Update on molecular imaging and precision medicine in lung cancer. Radiol Clin North Am. 2021;59(5):693-703. doi:10.1016/j.rcl.2021.05.002

4. Kim MJ, Yun GJ, Kim SE. Metabolic regulation of ferroptosis in cancer. Biology. 2021;10(2). doi:10.3390/biology10020083
5. Li S, Huang Y. Ferroptosis: an iron-dependent cell death form linking metabolism, diseases, immune cell and targeted therapy. Clin Transl Oncol. 2021. doi:10.1007/s12094-021-02669-8

6. Aguirre JI, Castillo EJ, Kimmel DB. Biologic and pathologic aspects of osteocytes in the setting of medication-related osteonecrosis of the jaw (MRONJ). Bone. 2021;153:116168. doi:10.1016/j.bone.2021.116168

7. Dixon SJ, Lemberg KM, Lamprecht MR, et al. Ferroptosis: an iron-dependent form of nonapoptotic cell death. Cell. 2012;149 (5):1060-1072. doi:10.1016/j.cell.2012.03.042

8. Kinowaki Y, Taguchi T, Onishi I, Kirimura S, Kitagawa M, Yamamoto K. Overview of ferroptosis and synthetic lethality strategies. Int J Mol Sci. 2021;22(17):9271. doi:10.3390/ijms22 179271

9. Mazhar M, Din AU, Ali H, et al. Implication of ferroptosis in aging. Cell Death Discov. 2021;7(1):149.

10. Li J, Cao F, Yin HL, et al. Ferroptosis: past, present and future. Cell Death Dis. 2020;11(2):88. doi:10.1038/s41419-020-2298-2

11. Lu R, Jiang Y, Lai X, Liu S, Sun L, Zhou ZW. A shortage of FTH induces ROS and sensitizes RAS-proficient neuroblastoma N2A cells to ferroptosis. Int J Mol Sci. 2021;22(16):8898. doi:10.3390/ijms 22168898

12. Yao $\mathrm{F}$, Cui $\mathrm{X}$, Zhang $\mathrm{Y}$, et al. Iron regulatory protein 1 promotes ferroptosis by sustaining cellular iron homeostasis in melanoma. Oncol Lett. 2021;22(3):657. doi:10.3892/ol.2021.12918

13. Chai M, Li X, Zhang Y, et al. A nomogram integrating ferroptosisand immune-related biomarkers for prediction of overall survival in lung adenocarcinoma. Front Genet. 2021;12:706814. doi:10.3389/ fgene. 2021.706814

14. Liu T, Yang Q, Zheng H, et al. Multifaceted roles of a bioengineered nanoreactor in repressing radiation-induced lung injury. Biomaterials. 2021;277:121103. doi:10.1016/j.biomaterials.2021.121103

15. Cao JY, Dixon SJ. Mechanisms of ferroptosis. Cell Mol Life Sci. 2016;73(11-12):2195-2209. doi:10.1007/s00018-016-2194-1

16. Latunde-Dada GO. Ferroptosis: role of lipid peroxidation, iron and ferritinophagy. Biochim Biophys Acta Gen Subj. 2017;1861 (8):1893-1900. doi:10.1016/j.bbagen.2017.05.019

17. Kajarabille N, Latunde-Dada GO. Programmed cell-death by ferroptosis: antioxidants as mitigators. Int $J$ Mol Sci. 2019;20:19. doi:10.3390/ijms20194968

18. Tu H, Tang LJ, Luo XJ, Ai KL, Peng J. Insights into the novel function of system Xc- in regulated cell death. Eur Rev Med Pharmacol Sci. 2021;25(3):1650-1662. doi:10.26355/eurrev_202102_24876

19. Sakakura Y, Sato H, Shiiya A, et al. Expression and function of cystine/glutamate transporter in neutrophils. J Leukoc Biol. 2007;81 (4):974-982. doi:10.1189/jlb.0606385

20. Miladinovic T, Ungard RG, Linher-Melville K, Popovic S, Singh G. Functional effects of TrkA inhibition on system xC(-)-mediated glutamate release and cancer-induced bone pain. Mol Pain. 2018;14:1744806918776467. doi:10.1177/1744806918776467

21. Ren Q, Sun RR, Zhao XF, Wang JX. A selenium-dependent glutathione peroxidase (Se-GPx) and two glutathione S-transferases (GSTs) from Chinese shrimp (Fenneropenaeus chinensis). Comp Biochem Physiol C Toxicol Pharmacol. 2009;149(4):613-623. doi:10.1016/j.cbpc.2009.01.007

22. Doll S, Freitas FP, Shah R, et al. FSP1 is a glutathione-independent ferroptosis suppressor. Nature. 2019;575(7784):693-698. doi:10.10 38/s41586-019-1707-0

23. Yang WS, Stockwell BR. Ferroptosis: death by lipid peroxidation. Trends Cell Biol. 2016;26(3):165-176. doi:10.1016/j.tcb.2015.10.014

24. Sun Y, Berleth N, Wu W, et al. Fin56-induced ferroptosis is supported by autophagy-mediated GPX4 degradation and functions synergistically with mTOR inhibition to kill bladder cancer cells. Cell Death Dis. 2021;12(11):1028. doi:10.1038/s41419-021-04306-2

25. Feng H, Stockwell BR. Unsolved mysteries: how does lipid peroxidation cause ferroptosis? PLoS Biol. 2018;16(5):e2006203. doi:10.1371/journal.pbio.2006203 
26. Yuan S, Wei C, Liu G, et al. Sorafenib attenuates liver fibrosis by triggering hepatic stellate cell ferroptosis via HIF-1alpha/SLC7A11 pathway. Cell Prolif;2021. e13158. doi:10.1111/cpr.13158

27. Luo X, Hill M, Johnson A, Latunde-Dada GO. Modulation of Deytb (Cybrd 1) expression and function by iron, dehydroascorbate and Hif-2alpha in cultured cells. Biochim Biophys Acta. 2014;1840 (1):106-112. doi:10.1016/j.bbagen.2013.08.012

28. Luo M, Tian R, Yang Z, Peng YY, Lu N. Quercetin suppressed NADPH oxidase-derived oxidative stress via heme oxygenase-1 induction in macrophages. Arch Biochem Biophys. 2019;671:69-76. doi:10.1016/j.abb.2019.06.007

29. Anderson GJ, Frazer DM, McKie AT, Wilkins SJ, Vulpe CD. The expression and regulation of the iron transport molecules hephaestin and IREG1: implications for the control of iron export from the small intestine. Cell Biochem Biophys. 2002;36(2-3):137-146. doi:10.13 85/CBB:36:2-3:137

30. Song Y, Wang B, Zhu X, et al. Human umbilical cord blood-derived MSCs exosome attenuate myocardial injury by inhibiting ferroptosis in acute myocardial infarction mice. Cell Biol Toxicol. 2021;37 (1):51-64. doi:10.1007/s10565-020-09530-8

31. Yang Q, Liu W, Zhang S, Liu S. The cardinal roles of ferroportin and its partners in controlling cellular iron in and out. Life Sci. 2020;258:118135. doi:10.1016/j.lfs.2020.118135

32. Guan W, Xia M, Ji M, et al. Iron induces two distinct $\mathrm{Ca}(2+)$ signalling cascades in astrocytes. Commun Biol. 2021;4(1):525. doi:10.1038/s42003-021-02060-x

33. Oosterheert W, van Bezouwen LS, Rodenburg RNP, et al. Cryo-EM structures of human STEAP4 reveal mechanism of iron(III) reduction. Nat Commun. 2018;9(1):4337. doi:10.1038/s41467-018-06817-7

34. Gao G, Li J, Zhang Y, Chang YZ. Cellular iron metabolism and regulation. Adv Exp Med Biol. 2019;1173:21-32.

35. Yan HF, Zou T, Tuo QZ, et al. Ferroptosis: mechanisms and links with diseases. Signal Transduct Target Ther. 2021;6(1):49. doi:10.1038/s41392-020-00428-9

36. Volani C, Doerrier C, Demetz E, et al. Dietary iron loading negatively affects liver mitochondrial function. Metallomics. 2017;9 (11):1634-1644. doi:10.1039/C7MT00177K

37. Liu J, Kang R, Tang D. Signaling pathways and defense mechanisms of ferroptosis. FEBS J. 2021. doi:10.1111/febs.16059

38. Dietrich C, Hofmann TG. Ferroptosis meets cell-cell contacts. Cells. 2021;10(9):2462. doi:10.3390/cells10092462

39. Han YK, Kim JS, Lee GB, Lim JH, Park KM. Oxidative stress following acute kidney injury causes disruption of lung cell cilia and their release into the bronchoalveolar lavage fluid and lung injury, which are exacerbated by Idh2 deletion. Redox Biol. 2021;46:102077. doi:10.1016/j.redox.2021.102077

40. Huang F, Yang R, Xiao Z, et al. Targeting ferroptosis to treat cardiovascular diseases: a new continent to be explored. Front Cell Dev Biol. 2021;9:737971. doi:10.3389/fcell.2021.737971

41. Li Q, Li QQ, Jia JN, et al. Baicalein exerts neuroprotective effects in $\mathrm{FeCl} 3$-induced posttraumatic epileptic seizures via suppressing ferroptosis. Front Pharmacol. 2019;10:638. doi:10.3389/fphar.2019. 00638

42. Lee H, Zhuang L, Gan B. Energy stress inhibits ferroptosis via AMPK. Mol Cell Oncol. 2020;7(4):1761242. doi:10.1080/23723 556.2020 .1761242

43. Sha W, Hu F, Xi Y, Chu Y, Bu S. Mechanism of ferroptosis and its role in Type 2 diabetes mellitus. J Diabetes Res. 2021;2021:9999612. doi:10.1155/2021/9999612

44. Tang Y, Zhou J, Hooi SC, Jiang YM, Lu GD. Fatty acid activation in carcinogenesis and cancer development: essential roles of long-chain acyl-CoA synthetases. Oncol Lett. 2018;16(2):1390-1396. doi:10.3892/ ol.2018.8843
45. Li Z, Ding T, Pan X, et al. Lysophosphatidylcholine acyltransferase 3 knockdown-mediated liver lysophosphatidylcholine accumulation promotes very low density lipoprotein production by enhancing microsomal triglyceride transfer protein expression. $J$ Biol Chem. 2012;287(24):20122-20131. doi:10.1074/jbc.M111.334664

46. Kagan VE, Mao G, Qu F, et al. Oxidized arachidonic and adrenic PEs navigate cells to ferroptosis. Nat Chem Biol. 2017;13(1):81-90. doi: $10.1038 /$ nchembio. 2238

47. Yang WS, Kim KJ, Gaschler MM, Patel M, Shchepinov MS, Stockwell BR. Peroxidation of polyunsaturated fatty acids by lipoxygenases drives ferroptosis. Proc Natl Acad Sci U S A. 2016;113(34): E4966-4975. doi:10.1073/pnas.1603244113

48. Orlando UD, Garona J, Ripoll GV, et al. The functional interaction between Acyl-CoA synthetase 4, 5-lipooxygenase and cyclooxygenase- 2 controls tumor growth: a novel therapeutic target. PLoS One. 2012;7(7):e40794. doi:10.1371/journal.pone.0040794

49. Shah R, Shchepinov MS, Pratt DA. Resolving the role of lipoxygenases in the initiation and execution of ferroptosis. ACS Cent Sci. 2018;4(3):387-396. doi:10.1021/acscentsci.7b00589

50. Chen P, Wu Q, Feng J, et al. Erianin, a novel dibenzyl compound in Dendrobium extract, inhibits lung cancer cell growth and migration via calcium/calmodulin-dependent ferroptosis. Signal Transduct Target Ther. 2020;5(1):51. doi:10.1038/s41392-020-0149-3

51. Muka T, Kraja B, Ruiter R, et al. Dietary mineral intake and lung cancer risk: the Rotterdam Study. Eur J Nutr. 2017;56(4):1637-1646. doi:10.1007/s00394-016-1210-4

52. Mahabir S, Forman MR, Dong YQ, Park Y, Hollenbeck A, Schatzkin A. Mineral intake and lung cancer risk in the NIH-American Association of Retired Persons Diet and Health study. Cancer Epidemiol Biomarkers Prev. 2010;19(8):1976-1983. doi:10.1158/1055-9965.EPI-10-0067

53. Mahabir S, Forman MR, Barerra SL, Dong YQ, Spitz MR, Wei Q. Joint effects of dietary trace metals and DNA repair capacity in lung cancer risk. Cancer Epidemiol Biomarkers Prev. 2007;16 (12):2756-2762. doi:10.1158/1055-9965.EPI-07-0324

54. Zhou W, Park S, Liu G, et al. Dietary iron, zinc, and calcium and the risk of lung cancer. Epidemiology. 2005;16(6):772-779. doi:10.1097/ 01.ede.0000181311.11585.59

55. Mahabir S, Wei Q, Barrera SL, et al. Dietary magnesium and DNA repair capacity as risk factors for lung cancer. Carcinogenesis. 2008;29(5):949-956. doi:10.1093/carcin/bgn043

56. Walter PB, Knutson MD, Paler-Martinez A, et al. Iron deficiency and iron excess damage mitochondria and mitochondrial DNA in rats. Proc Natl Acad Sci U S A. 2002;99(4):2264-2269. doi:10.1073/ pnas. 261708798

57. Altieri F, Grillo C, Maceroni M, Chichiarelli S. DNA damage and repair: from molecular mechanisms to health implications. Antioxid Redox Signal. 2008;10(5):891-937. doi:10.1089/ars.2007.1830

58. Wei Q, Cheng L, Amos CI, et al. Repair of tobacco carcinogen-induced DNA adducts and lung cancer risk: a molecular epidemiologic study. J Natl Cancer Inst. 2000;92(21):1764-1772. doi:10.1093/jnci/92.21.1764

59. Ohno M, Miura T, Furuichi M, et al. A genome-wide distribution of 8-oxoguanine correlates with the preferred regions for recombination and single nucleotide polymorphism in the human genome. Genome Res. 2006;16(5):567-575. doi:10.1101/gr.4769606

60. Shibata Y, Yasui H, Higashikawa K, Miyamoto N, Kuge Y. Erastin, a ferroptosis-inducing agent, sensitized cancer cells to X-ray irradiation via glutathione starvation in vitro and in vivo. PLoS One. 2019;14 (12):e0225931. doi:10.1371/journal.pone.0225931

61. Chen G, Fillebeen C, Wang J, Pantopoulos K. Overexpression of iron regulatory protein 1 suppresses growth of tumor xenografts. Carcinogenesis. 2007;28(4):785-791. doi:10.1093/carcin/bgl210 
62. Chen H, Zheng C, Zhang Y, Chang YZ, Qian ZM, Shen X. Heat shock protein 27 downregulates the transferrin receptor 1-mediated iron uptake. Int $J$ Biochem Cell Biol. 2006;38(8):1402-1416. doi:10.1016/j.biocel.2006.02.006

63. Baek S, Choi CM, Ahn SH, et al. Exploratory clinical trial of (4S)-4-(3-[18F]fluoropropyl)-L-glutamate for imaging $\mathrm{xC}$ - transporter using positron emission tomography in patients with non-small cell lung or breast cancer. Clin Cancer Res. 2012;18(19):5427-5437. doi:10.1158/1078-0432.CCR-12-0214

64. Liu Y, Fan X, Zhao Z, Shan X. LncRNA SLC7A11-AS1 contributes to lung cancer progression through facilitating TRAIP expression by inhibiting miR-4775. Onco Targets Ther. 2020;13:6295-6302. doi:10.2147/OTT.S253082

65. Chen M, Jiang Y, Sun Y. KDM4A-mediated histone demethylation of SLC7A11 inhibits cell ferroptosis in osteosarcoma. Biochem Biophys Res Commun. 2021;550:77-83. doi:10.1016/j.bbrc.2021.02.137

66. Lou JS, Zhao LP, Huang ZH, et al. Ginkgetin derived from Ginkgo biloba leaves enhances the therapeutic effect of cisplatin via ferroptosis-mediated disruption of the $\mathrm{Nrf} / \mathrm{HO}-1$ axis in EGFR wild-type non-small-cell lung cancer. Phytomedicine. 2021;80:153370. doi:10.1016/j.phymed.2020.153370

67. Huang C, Yang M, Deng J, Li P, Su W, Jiang R. Upregulation and activation of $\mathrm{p} 53$ by erastin induced reactive oxygen species contribute to cytotoxic and cytostatic effects in A549 lung cancer cells. Oncol Rep. 2018;40(4):2363-2370. doi:10.3892/ or.2018.6585

68. Hu K, Li K, Lv J, et al. Suppression of the SLC7A11/glutathione axis causes synthetic lethality in KRAS-mutant lung adenocarcinoma. J Clin Invest. 2020;130(4):1752-1766. doi:10.1172/JCI124049

69. Zhang J, Liu Y, Guo Y, Zhao Q. GPX8 promotes migration and invasion by regulating epithelial characteristics in non-small cell lung cancer. Thorac Cancer. 2020;11(11):3299-3308. doi:10.1111/ 1759-7714.13671

70. Wang Z, Zhang X, Tian X, et al. CREB stimulates GPX4 transcription to inhibit ferroptosis in lung adenocarcinoma. Oncol Rep. 2021;45(6). doi:10.3892/or.2021.8039

71. Deng SH, Wu DM, Li L, et al. miR-324-3p reverses cisplatin resistance by inducing GPX4-mediated ferroptosis in lung adenocarcinoma cell line A549. Biochem Biophys Res Commun. 2021;549:54-60. doi:10.1016/j.bbrc.2021.02.077

72. Tang X, Ding H, Liang M, et al. Curcumin induces ferroptosis in non-small-cell lung cancer via activating autophagy. Thorac Cancer. 2021;12(8):1219-1230. doi:10.1111/1759-7714.13904

73. Meng M, Huang M, Liu C, et al. Local anesthetic levobupivacaine induces ferroptosis and inhibits progression by up-regulating p53 in non-small cell lung cancer. Aging. 2021;13. doi:10.18632/ aging. 203138

74. Chen YM, Lai CH, Lin CY, et al. Body mass index, weight loss, and mortality risk in advanced-stage non-small cell lung cancer patients: a focus on EGFR mutation. Nutrients. 2021;13(11):3761. doi:10.3390/nu13113761

75. Zhang T, Sun B, Zhong C, et al. Targeting histone deacetylase enhances the therapeutic effect of Erastin-induced ferroptosis in EGFR-activating mutant lung adenocarcinoma. Transl Lung Cancer Res. 2021;10(4):1857-1872. doi:10.21037/tlcr-21-303

76. Lee CK, Park KK, Chung AS, Chung WY. Ginsenoside Rg3 enhances the chemosensitivity of tumors to cisplatin by reducing the basal level of nuclear factor erythroid 2-related factor 2-mediated heme oxygenase-1/NAD(P)H quinone oxidoreductase-1 and prevents normal tissue damage by scavenging cisplatin-induced intracellular reactive oxygen species. Food Chem Toxicol. 2012;50 (7):2565-2574. doi:10.1016/j.fct.2012.01.005
77. Li $\mathrm{X}, \mathrm{Mu} \mathrm{J}$, Lin $\mathrm{Y}$, Zhao J, Meng $\mathrm{X}$. Combination of cyanidin-3-O-glucoside and cisplatin induces oxidative stress and apoptosis in HeLa cells by reducing activity of endogenous antioxidants, increasing bax/bcl-2 mRNA expression ratio, and downregulating Nrf2 expression. $J$ Food Biochem. 2021;45(7):e13806. doi:10.1111/jfbc.13806

78. Tang Z, Jiang W, Mao M, Zhao J, Chen J, Cheng N. Deubiquitinase USP35 modulates ferroptosis in lung cancer via targeting ferroportin. Clin Transl Med. 2021;11(4):e390. doi:10.1002/ctm2.390

79. Li Y, Yan H, Xu X, Liu H, Wu C, Zhao L. Erastin/sorafenib induces cisplatin-resistant non-small cell lung cancer cell ferroptosis through inhibition of the Nrf2/xCT pathway. Oncol Lett. 2020;19(1):323-333.

80. Horibe S, Kawauchi S, Tanahashi T, Sasaki N, Mizuno S, Rikitake Y. $\mathrm{CD} 44 \mathrm{v}$-dependent upregulation of $\mathrm{xCT}$ is involved in the acquisition of cisplatin-resistance in human lung cancer A549cells. Biochem Biophys Res Commun. 2018;507(1-4):426-432. doi:10.1016/j.bbrc.2018.11.055

81. Huang Y, Dai Z, Barbacioru C, Sadee W. Cystine-glutamate transporter SLC7A11 in cancer chemosensitivity and chemoresistance. Cancer Res. 2005;65(16):7446-7454. doi:10.1158/0008-5472.CAN-04-4267

82. Lei G, Zhang Y, Koppula P, et al. The role of ferroptosis in ionizing radiation-induced cell death and tumor suppression. Cell Res. 2020;30(2):146-162. doi:10.1038/s41422-019-0263-3

83. Wang W, Green M, Choi JE, et al. CD8(+) T cells regulate tumour ferroptosis during cancer immunotherapy. Nature. 2019;569 (7755):270-274. doi:10.1038/s41586-019-1170-y

84. Kong R, Wang N, Han W, Bao W, Lu J. IFNgamma-mediated repression of system $\mathrm{xc}(-)$ drives vulnerability to induced ferroptosis in hepatocellular carcinoma cells. $J$ Leukoc Biol. 2021;110 (2):301-314. doi:10.1002/JLB.3MA1220-815RRR

85. Wang S, Chen S, Ying Y, et al. Comprehensive analysis of ferroptosis regulators with regard to PD-L1 and immune infiltration in clear cell renal cell carcinoma. Front Cell Dev Biol. 2021;9:676142. doi:10.3389/fcell.2021.676142

86. Galvan I, Inacio A, Danino M, Corbi-Llopis R, Monserrat MT, Bernabeu-Wittel J. High SLC7A11 expression in normal skin of melanoma patients. Cancer Epidemiol. 2019;62:101582. doi:10.1016/j.canep.2019.101582

87. Zhou W, Zhang J, Yan M, et al. Orlistat induces ferroptosis-like cell death of lung cancer cells. Front Med. 2021. doi:10.1007/s11684-020-0804-7

88. Yao X, Yang P, Jin Z, et al. Multifunctional nanoplatform for photoacoustic imaging-guided combined therapy enhanced by $\mathrm{CO}$ induced ferroptosis. Biomaterials. 2019;197:268-283. doi:10.1016/j. biomaterials.2019.01.026

89. Hu S, Ma J, Su C, et al. Engineered exosome-like nanovesicles suppress tumor growth by reprogramming tumor microenvironment and promoting tumor ferroptosis. Acta Biomater. 2021;135:567-581. doi:10.1016/j.actbio.2021.09.003

90. Song R, Li T, Ye J, et al. Acidity-activatable dynamic nanoparticles boosting ferroptotic cell death for immunotherapy of cancer. $A d v$ Mater. 2021;33(31):e2101155. doi:10.1002/adma.202101155

91. Hsieh $\mathrm{CH}$, Hsieh HC, Shih FS, et al. An innovative NRF2 nano-modulator induces lung cancer ferroptosis and elicits an immunostimulatory tumor microenvironment. Theranostics. 2021;11 (14):7072-7091. doi:10.7150/thno.57803

92. de Souza MGF, de Jesus Guedes FN, Tebaldi ML, et al. Ferriliposomes: preformulation and selective cytotoxicity against A549 lung cancer cells. Pharmaceutics. 2021;13(5):712. doi:10.3390/ pharmaceutics 13050712

93. Gai C, Liu C, Wu X, et al. MT1DP loaded by folate-modified liposomes sensitizes erastin-induced ferroptosis via regulating miR-365a-3p/NRF2 axis in non-small cell lung cancer cells. Cell Death Dis. 2020;11(9):751. doi:10.1038/s41419-020-02939-3 


\section{Publish your work in this journal}

The Journal of Inflammation Research is an international, peerreviewed open-access journal that welcomes laboratory and clinical findings on the molecular basis, cell biology and pharmacology of inflammation including original research, reviews, symposium reports, hypothesis formation and commentaries on: acute/chronic inflammation; mediators of inflammation; cellular processes; molecular mechanisms; pharmacology and novel anti-inflammatory drugs; clinical conditions involving inflammation. The manuscript management system is completely online and includes a very quick and fair peerreview system. Visit http://www.dovepress.com/testimonials.php to read real quotes from published authors. 